\title{
Wavelet Analysis in Video Watermarking through Wavelet Transform Scheme
}

\author{
Gunjan Malik \\ M.Tech, Computer Science \\ Engineering, The NorthCap \\ University, Haryana \\ India
}

\author{
Tarun Kumar \\ Lecturer \\ Government Polytechnic \\ College Amroha, UP \\ India
}

\author{
Gaurav Agarwal \\ Astt. Professor. \\ The NorthCap University, \\ Haryana \\ India
}

\begin{abstract}
Intellectual and copyright protection is one of the major issues faced by copyright owners. Easy access to Internet and all the digital media such as audios, images, digital documents and videos, poses great threat to copyright owners as their work gets manipulated, forged, redistributed conveniently through illegal means. As an effective solution to this problem, concept of Digital Watermarking has been used. Watermarks can be of the form images, text, binary logos, signatures, and numbers. They are used for storing information about the copyright owner, source of data, and authentic users. In the proposed work, video watermarking technique has been shown highlighting comparative analysis of db wavelets based on different quality parameters. Each of the $\mathrm{db}$ wavelets is applied on the randomly selected frames from the input coloured video using random number that works as a key for the proposed extraction algorithm. It is shown that not all $\mathrm{db}$ wavelets support watermarking scheme. Out of 45 wavelets, $12 \mathrm{db}$ wavelets were applicable for watermarking. The original watermark image and the extracted watermark image are then used as the basis against various quality parameters to check if the imperceptibility of the watermark is retained after watermark extraction. The proposed watermarking scheme is imperceptible against various quality parameters such as Peak-signal-to-noise ratio, Mean-square error, maximum difference, and normalized absolute error.
\end{abstract}

\section{Keywords}

Blind, Non-Blind Watermarks, Fidelity, Robustness, Spatial domain, Frequency Domain, DFT, DWT, DCT, PCA, Wavelet Packet Transform, Arnold Transform, Db wavelet, Synchronous and asynchronous attacks.

\section{INTRODUCTION}

Internet environment is open for all. With the widespread use of Internet facility, anyone can have easy access to digitized multimedia such as videos, audios, documents and images. Internet downloading is also easily achievable. But this give rise to problems in copyright protection as it enhances illegal copying, and distribution of copyright content at large. Digitized content in the form of images, audios, text, files and videos can be easily copied, moved, modified, redistributed which can also be referred to as Forgery of data. This is huge threat to copyright owners as their work gets manipulated and distributed. This leads to decline in their profitability. Also, anyone can claim its authority and ownership if the information about the actual owner of data is missing. Hence, to overcome such copyright and intellectual right protection issues, Digital Watermarking emerged as a useful solution [1]. It is different from the techniques of Cryptographic encryption/decryption as the data in encrypted form becomes non-readable and will not be of any use if users desire to view that data. Through the technique of watermarking, useful information such as author's details, information about the legal users of the content is embedded into the digitized content known as watermarks [1]. This helps in preventing claiming of authorship from some third party illegally. Apart from copy control, tamper detection, copyright protection, authentication, fingerprinting [9], watermarks can also be applied to applications such as data integrity verification, broadcast monitoring, Embedding $\mathrm{QR}$ codes for quality monitoring [7], image indexing and labelling, and embedding hospital logos in medical imagery [8] [2] .

Though Digital watermarking is mature field of research; however, due to rapidly changing technology, newer applications poses new challenges which require constant effort to enhance performance [2]. One such challenge is to make the watermark robust enough to withstand consecutive attacks. Online sharing of multimedia such as videos, MMS clips, images on social sites such as Facebook, Instagram and other mediums can possibly render the original data to be subjected to several processing such as resizing, contrast adjustment, compressions, blurring, etc. Thus the embedded watermark should have to be robust against them.

\subsection{Characteristics Of Watermark}

Watermarks are codes which can be in form of logo, fingerprints, text, binary data, numerical data, audio signals, images or can be signatures. A watermarking scheme is expected to possess three main characteristics: High Fidelity, Good Robustness, and High Data Capacity [3]. Fidelity implies that the watermarked image should be as perceptually equal as possible to the original host image. Withstanding several processing attacks, yet the information stored inside the watermark remains intact and can be extracted successfully, leads to its robustness. The more the capacity, more private information can be embedded inside the original host image. However, it is difficult to achieve all characteristics in one watermarking scheme as focusing on fidelity may lead to decrease in robustness, or striving to store more information inside the image may hamper the image quality. Watermarks must be embedded in the host content in such a manner that it becomes difficult to remove and perceive [4].

\subsection{Types of Watermark And \\ Watermarking Scheme}

One set of characterization of watermarking scheme is blind, semi-blind and non-blind [4]. Non-blind watermarks require the presence of host image at the time of watermark extraction. Though it is secure but this limitation makes it less used. Semi-blind require the presence of only the secret key and the watermark at the time of extraction. Blind watermarks 
are most used as they do not require the prior presence of the host image for watermark extraction.

Watermarks can be visible or non-visible in nature [5]. Visible watermark are generally used to put logo on the host data. They can be seen by human eye. Invisible watermark is inserted invisibly inside the original host data. Invisible watermarks can be further of Fragile or Robust type in nature. Fragile watermark is generally added inside digital media with the motive to detect if some attack was made on data to damage the watermark; on the other hand, robust watermark is robust to different attacks retaining the information inside the watermark even after attack [6].

Watermarking can be of different categories: Text watermarking, Image watermarking, Audio watermarking, Video watermarking. For video watermarking, the main concern is to apply watermark on video streams either in compressed form or uncompressed. Generally, videos in mobiles or other electronic devices are present in already compressed format (such as .mgp, .flv, .mp3, etc.). The video watermarking technique has to be compatible to both formats. Digital videos can be widely utilized in applications such as wireless videos, video on demand, video broadcasting, in entertainment industry, video phone and many others [10].

\subsection{Watermarking Domains}

Since, the start of digital watermarking technique as an efficient solution to intellectual rights and copyright protection, several different methods and approaches were proposed. Some deal in spatial domain, while others use frequency domain. Watermarking can be template based or content based as well. Content based watermarking technique can be found in [11].

\subsubsection{Spatial Domain}

Watermark is embedded in spatial domain by modifying the pixel values of the host image. Two commonly used methods in spatial domain are: Least Significant Bit and Correlation based techniques. It is conceptually simple domain for watermarking schemes. Also, involve less computational intricacies, suitable for video watermarking where prime goal is real time performance and robustness can be made secondary. However, it suffers from hostile attacks and other signal compression [1]. They are comparatively weak to intensity adjustments [12], filtering, and perceptual tuning [4].

\subsubsection{Transform Domain}

In frequency domain, transform coefficients are adjusted for watermark embedding. It is used widely as compared to spatial domain as it yields more robust results to geometrical transformations such as translation, scaling, bending, shearing, rotation [4] and compressions [1]. It also helps maintain the imperceptibility of original host image. Common transforms used for watermarking are, discrete Fourier transform, discrete cosine transform, discrete wavelet transform, wavelet packet transform, principle component analysis which is a linear transform.

\section{Table 1. Comparison Between Spatial Domain And} Frequency Domain [6]

\begin{tabular}{|c|c|}
\hline Spatial Domain & Frequency Domain \\
\hline $\begin{array}{c}\text { Simple technique to use by } \\
\text { modifying pixel values }\end{array}$ & $\begin{array}{c}\text { Complex to use by } \\
\text { modifying transform } \\
\text { coefficients }\end{array}$ \\
\hline Lack Imperceptibility & High imperceptibility \\
\hline $\begin{array}{c}\text { Less robust, incompetence } \\
\text { in dealing with a range of }\end{array}$ & $\begin{array}{c}\text { More robust against } \\
\text { different types of attacks }\end{array}$ \\
\hline
\end{tabular}

\begin{tabular}{|c|c|}
\hline attacks & \\
\hline $\begin{array}{l}\text { Restricted capacity of an } \\
\text { image to hold the } \\
\text { watermark }\end{array}$ & $\begin{array}{l}\text { High capacity to hold } \\
\text { watermark }\end{array}$ \\
\hline $\begin{array}{l}\text { Involves less computational } \\
\text { cost }\end{array}$ & $\begin{array}{l}\text { High computational cost } \\
\text { involved }\end{array}$ \\
\hline $\begin{array}{l}\text { Poor performance suitable } \\
\text { only for video } \\
\text { watermarking where video } \\
\text { quality is given priority } \\
\text { over robustness to attacks. }\end{array}$ & $\begin{array}{l}\text { Better performance and } \\
\text { results }\end{array}$ \\
\hline
\end{tabular}

\section{LITERATURE SURVEY}

Research in the field of Digital Watermarking gained attention in early to mid-1990s. Since then, it has gained wide exposure and many different techniques have been proposed with the common goal to achieve high robustness and imperceptibility facing newer challenges.

Qianli [1], proposed the algorithm of DWT and DCT to achieve watermarking, thereby enhancing robustness. To protect the copyright information of digital media efficiently, initially, the grayscale image is taken as input which is first transformed into discrete wavelet components up to 3 levels. Then, to each sub block, discrete cosine transform is applied. The watermarked image is also transformed into discrete cosine components and thus embedded into the host image. The Watermark extraction algorithm proposed was the reverse of the transforms to achieve the watermark. The experimental results showcased that algorithms applied yielded robust outcome when the watermark was subjected to lossy compressions, low pass filtering, cutting, and noise.

Hassen [4], has proposed the use of Schur transformation for the watermarking scheme. The method proposed is robust against many attacks both asynchronous attacks such as geometric attacks and synchronous attacks such as filtering, noise addition, or compression. In this, blind watermark is used for embedding; hence, there will be no requirement of the host image for watermark detection and recovery process. In this, initially, the original image is transformed into schur domain which is represented in form of a triangular matrix. Then, non-sensitive zone is identified by carrying out tests where the watermark can be suitably embedded keeping the image quality unaffected. Lowest values of the matrix are processed so that as lowest distortion on watermarked image is possible. Experimental results showed that the proposed method was robust more than spatial method or DCT, DFT.

DWT-SVD based watermarking scheme using Arnold transform has been proposed in paper [13], Jeril proposed a novel color image watermarking using level-3 DWT, followed by SVD of the LH sub band of the cover image and applying SVD to the scrambled watermark. Singular values of the cover image are modified to embed the scrambled logo. Algorithm presented involves semi-blind watermark extraction which avoids the need for original image during extraction process. The watermarking system gives good imperceptibility and robustness against various image processing attacks. Arnold transform is used to cater to the need of watermark security as the intention is that the watermark after extraction should be visible only to the authorized and authenticated users. Hence, a secret key is shared between the owner and intended users.

HVS characteristics along with Spread Transform has been proposed in [14] for watermarking scheme. Already known spread based transformations use watermark signal with real number sequences which were not able to have unique 
signature and hence the use of spread watermark according to Watson Model to convey unique information with great resiliency is proposed in this paper. Watermark image is firstly spatially dispersed and then hadamard transformation is applied on cover \& dispersed watermark image. After sorting out the transform coefficients of the cover and the watermark image in ascending order, apply the modular function obtained from the application of Watson visual and exponential function of entropy masking model and then block based inverse Hadamard transform is applied to form watermarked image. This scheme is showing resiliency even after embedding of watermark multiple times to the original image.

Embedding watermark can be considered as an optimization problem. With this viewpoint, Yueh-Hong Chen [3], proposed GA based image watermarking technique using wavelet packet transform. GA is applied in frequency domain to find out the optimal coefficients that can be manipulated for watermark embedding. Wavelet packet decomposition allows decompositions to be represented by any of permissible base represented by the subtree and with the use of genetic algorithm it allows to select most apt base to increase robustness. For embedding watermark, genetic algorithm is being used to find out the optimal base of WPT and random chosen coefficients are being used. Genetic algorithm is applied by selecting the parameters, fitness function and fitness values and having proper mutation and mate ratio to get the optimal value in each iteration of the evaluation while coefficients are being used in the manner if first watermark bit is 1 then all other coefficient should be smaller than first coefficient and if it's 0 then all the coefficients are greater than 0. Extraction of the watermark involves the comparison of the first coefficient with largest and smallest from the remaining coefficients.

Mehran [2] proposed the technique of grayscale watermark logo embedding inside the host image using texturization scheme. In this, initially the host image is separated into fairly textured and poorly textured regions. Then, the watermark logo is converted into similar texture using Arnold transform and one lossless rotation technique to map with fairly textured image regions. Whereas, for the poorly textured regions, only lossless rotation is performed on the watermark logo. The watermark is then embedded into the host image regions using wavelet based embedding techniques.

In paper [11], blind image watermarking scheme is proposed in DFT domain. The scheme identifies phase features from low to middle band coefficients in DFT and then, Radon transformation is applied in order to fetch rotation and scaling values of the attack.

A hybrid combination of both DWT and PCA is proposed in [15-16]. PCA helps in providing complete decorrelation among coefficients and enhance data hiding. In [15], binary watermarks are embedded in video frames. The host video is first decomposed into sub bands using 3 level DWT, then, after applying entropy to each block, some blocks are selected on which PCA is applied. Then, the watermark is embedded into the highest coefficient values of PCA blocks. The algorithm showed high robustness and high imperceptibility when subjected to several attacks.

DCT based video watermarking technique for embedding blind watermark is proposed by Yanget al. in [17]. In this, firstly each of the video frames is sub divided into multiple blocks which get to formed using DCT technique. Then the watermark is embedded inside the uncompressed frame components into the luminance parts of the cover data in order to avoid chrominance quality distortion. The secret key is created which can be further used for watermark extraction.

\section{TRANSFORMS USED IN WATERMARKING SCHEME 3.1 DFT}

Discrete Fourier Transform converts continuous functions into frequency components. It only imparts frequency information without location updates [12]. It is full video/image transform, implying that any changes made to transform coefficients will affect the entire video/image [6]. This technique gives robust results against geometric attacks like cropping, scaling, and translation.

\subsection{DCT}

It transforms the signals into corresponding frequency components. It has the advantage of concentrating energy in lower order frequency coefficients [17]. It is greatly used in image compression techniques. Low frequencies hold large DCT coefficients while high frequencies hold small DCT coefficients. Thus, most of image energy is concentrated in low frequency regions [1]. Mainly, middle frequency bands are focused by DCT. It yields faster results as it takes complexity of the order $\mathrm{O}(\mathrm{n} \log \mathrm{n})$. It can withstand attacks like sharpening, blurring, filtering but unlike DWT it lack in imperceptibility as it cannot be modelled as HVS. Applying mid-band frequency can provide better trade-off between imperceptibility and robustness [6]. Real time applications of DCT are pan card, employee e-card, or fingerprint identity.

\subsection{DWT}

DWT technique is used in improving imperceptibility of the image. It is a multiresolution decomposition technique. It has space frequency localization property. If there is $1 \mathrm{D}$ signal, then, after applying Daubechies (db) wavelet transform, 8 different non-overlapping sub bands of multiple resolutions are created such as $\mathrm{db} 1, \mathrm{db} 2 \mathrm{uptil} \mathrm{db} 8$. Those sub bands can be further processed to achieve next level of wavelet coefficients [17]. It is found that db6; the 6th sub band signal represents best the original signal. Applying DWT on 2D signal, decomposes the signal into 4 non-overlapping sub bands, LL (approximation band), HL (horizontal), LH (vertical), HH (diagonal). The LL sub band can be further decomposed to a certain level as shown in Figure 1. LL band is happened to store most of the signal energy and can be thus used for watermark embedding. DWT can be precisely modelled to HVS system enabling embedding of watermark in those regions which are less sensitive to human vision. Applying watermark in high frequency band is less robust but leads to high fidelity, while in low band, better robustness can be achieved but at the cost of notable alteration to original image. Wavelet transform family comprises of a list of transforms, such as, Haar wavelet, Db wavelets, Coiflets, Symlets, Gaussian, Morlet, Meyer, DMeyer, Shannon and many more. Some have been described in following section. They can be of orthogonal type, biorthogonal, with or without scalar function or complex wavelets. Real time applications of DWT are military applications, government applications, and bank applications or broadcast monitoring. 


\begin{tabular}{|c|c|c|c|}
\hline LL3 & HL3 & HL2 & \\
\cline { 1 - 1 } LH3 & HH3 & HL1 \\
\cline { 1 - 1 } LH2 & HH2 & \\
\cline { 1 - 1 } & LH1 & HH1 \\
& & \\
\hline
\end{tabular}

Figure 1: Three level image decomposition using DWT

\subsection{Arnold Transform}

It helps in enhancing the security issues of the watermark. It has the property of scrambling. The watermark logo after subjected to Arnold transform can be scrambled. In this method, the image is hits truck with transformation which apparently randomizes the original arrangement of pixels [12]. On sufficiently number of iterations, the original image can be reconstructed. Any one of the scrambled logo can be chosen as secret key. To regain the meaningful logo from the extracted watermark, this secret key is used. Thus, watermark security can be ensured by using secret key which is known only to the content owner and authentic users.

\subsection{WPT}

Wavelet Packet Transform can be considered as a generalized version of wavelet transform [3]. It allows decompositions to be represented by any of permissible base represented by the subtree. It adopts redundant basis function and provide timefrequency resolution.

\subsection{Principle Component Analysis}

Apart from DCT, DFT, DWT techniques of frequency domain, PCA transform is also another technique that can be used for watermarking. This technique was first applied to gray scale image used in image watermarking. PCA helps in providing complete decorrelation among coefficients and enhance data hiding. PCA is used as it helps identify the components which are best suitable for watermark embedding.

\subsection{DB Wavelets Transform}

It constitutes another set of wavelet family comprising of 45 wavelets as $d b 1, d b 2$, up till db45. The $d b 1$ wavelet is represented similarly to Haar wavelet. They are orthogonal in nature. A scaling function is given to each $\mathrm{db}$ wavelet that can be used for analysis of orthogonal multiresolution. Db wavelets can be defined by evaluating the differences and mean of scalar products with scaling wavelet and signal. Db wavelet transforms are highly utilized in compression applications. Also helps eliminating noise from the digitized multimedia like videos, audio and images. They can be utilized in areas like image enhancement as well as signal recognition.

\section{PROPOSED WORK}

In the proposed watermarking method, a coloured watermark image is embedded into a video strip using db wavelets from discrete wavelet transformation family in frequency domain. The proposed algorithm is described below.

\subsection{Watermark Embedding Algorithm}

The embedding procedure involves various steps as mentioned below.

Step1: Read the host video and divide the video into frames.

Step2: Split each of the frame into Red, Green and Blue components.

Step3: Convert the RGB components of each of the frames into Grey components.

Step4: Apply random number generator to select random set of frames for watermark embedding.

Step5: Apply discrete wavelet transformation using $\mathrm{db}$ wavelets to each of the randomly selected frames in order to obtain four frequency sub bands, LL1, HL1, LH1 and HH1.

Step6: Now take the LL1 frequency sub band and apply second level DWT to obtain LL2, HL2, LH2 and HH2.

Step7: Again take LL2 frequency sub band and apply third level DWT to obtain LL3, HL3, LH3 and HH3.

Step8: Take the watermark image which is to be embedded in the host video frames and split it into red, blue and green components.

Step9: Convert the RGB components of the watermark image into grey component.

Step10: Apply wavelet transformation using db wavelets upto three level decomposition to obtain sub-bands LL3, HL3, LH3 and $\mathrm{HH} 3$.

Step11: The watermark bits are then embedded into significant coefficients of the host video frames.

Step12: Apply Inverse DWT to obtain the watermarked frames.

Step13: Reconstruct the watermarked video by combining frames. 


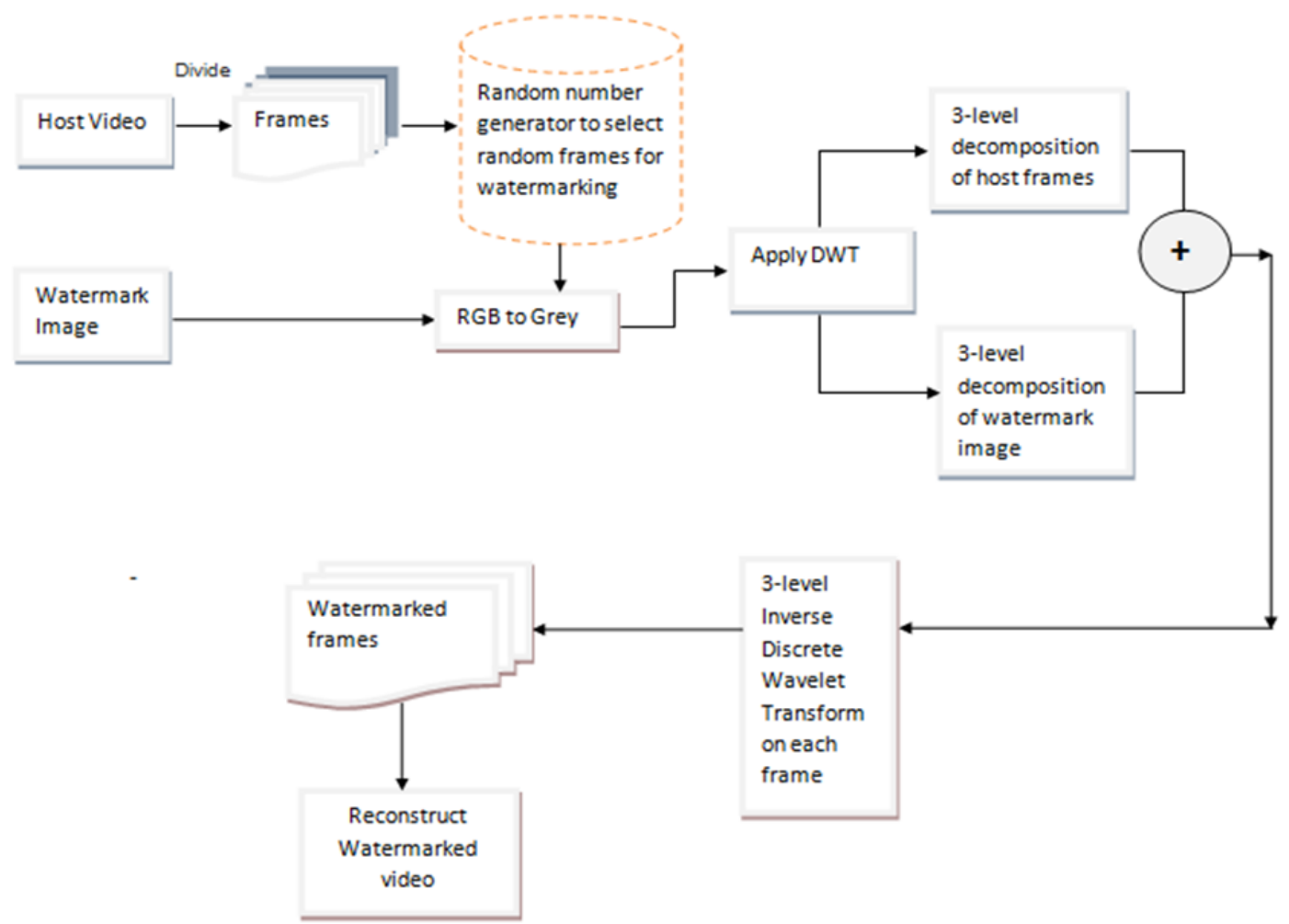

Figure 2. Watermark Embedding Algorithm

\subsection{Watermark Extraction Algorithm}

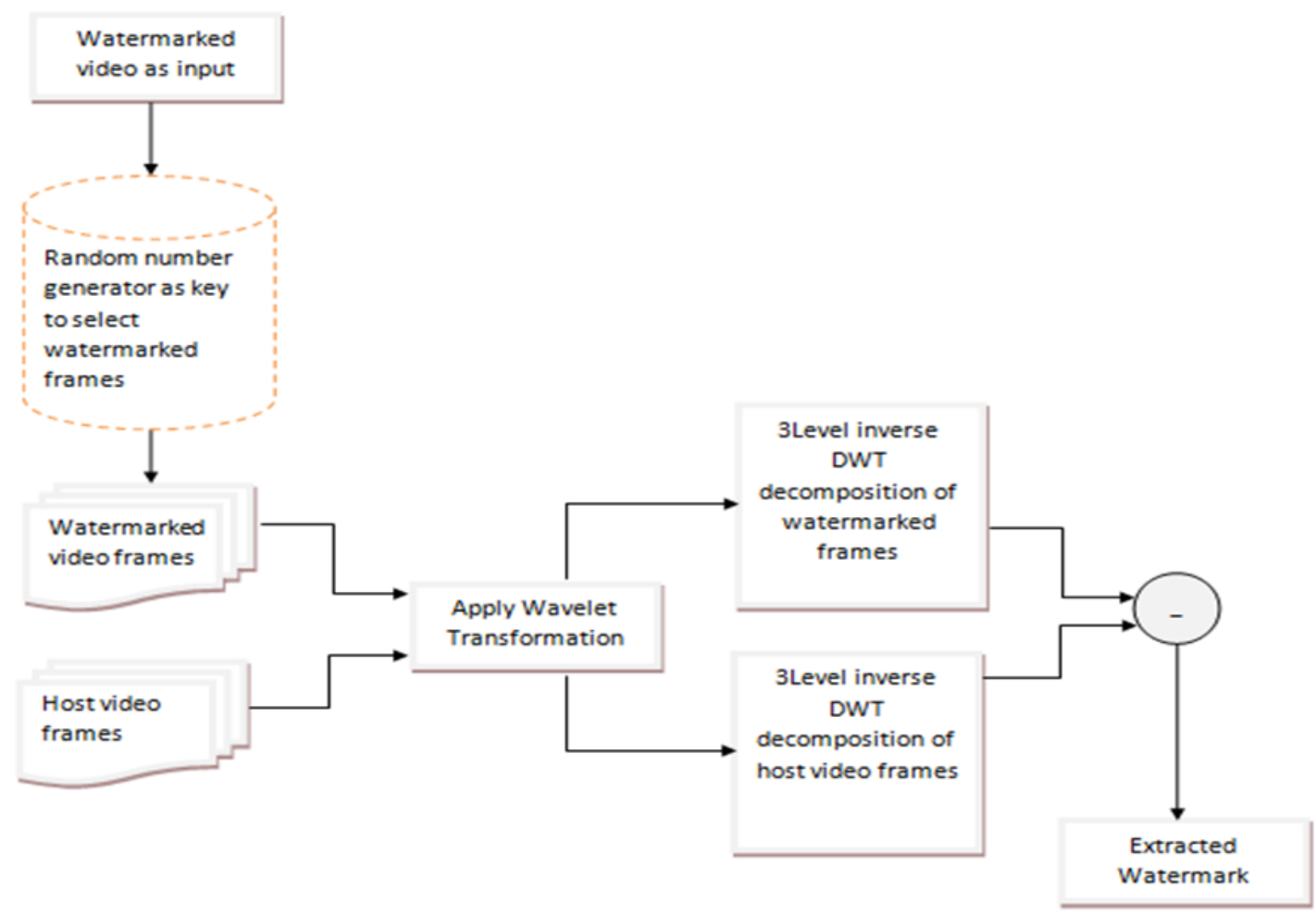

Figure 3. Watermark Extraction Algorithm

The extraction procedure involves extracting the watermark from the host video in which it was embedded. Various steps are involved which are as mentioned below.
Step2: Convert the red, blue and green components into grey component

Step1: Read the watermarked video and split it into frames 
Step3: Apply wavelet transformation using db wavelets upto three level decomposition to obtain sub-bands LL3, HL3, LH3 and $\mathrm{HH} 3$

Step4: Evaluate the difference between the LL3 frequency sub band of watermarked frame and the third level decomposed LL3 frequency sub band of the host frame to get the watermark.

\section{EXPERIMENTAL RESULTS}

In the project work, the experiments are carried using Matlab coding. We have prepared a GUI layout with a list of menus. Clicking on each menu will perform an independent function. The inputs used are a coloured video of avi type and a coloured watermark image that will be embedded inside the video frames.

All the frames are of same size. The watermark image used is of the same size of rows and columns as the video frames. The proposed technique uses a random number generator that generates a set of numbers that will be used as a secret key at the time of watermark extraction. Those numbers will be used as the references for selecting the random frames that will be further used for watermark embedding.

The proposed algorithm is executed for each of the $\mathrm{db}$ wavelet from $\mathrm{db} 1 \mathrm{to} \mathrm{db} 45$. It is observed that $12 \mathrm{db}$ wavelets support the watermarking scheme, namely $\mathrm{db} 45, \mathrm{db} 41, \mathrm{db} 37, \mathrm{db} 33$, db29, db25, db21, db17, db13, db9, db5, db1. The watermark image and the extracted watermark image are taken as input to various quality parameters.

The original host video and watermark image used is shown below:

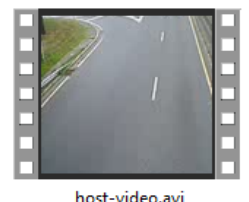

(a) Host video

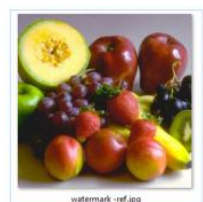

(b) Watermark image

After 3 level dwt applied on randomly selected frames, the output is shown below:
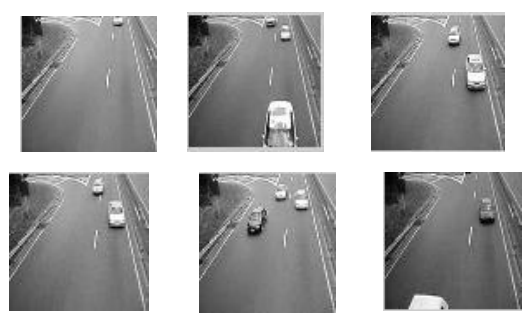

Figure 4. 3 level decomposition of host video frames

After 3 level dwt applied on watermarked image, the output is shown below:

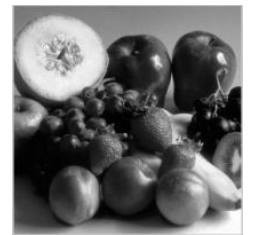

Figure 5. 3 level decomposition of watermark image
The frames obtained after embedding watermark is shown below:
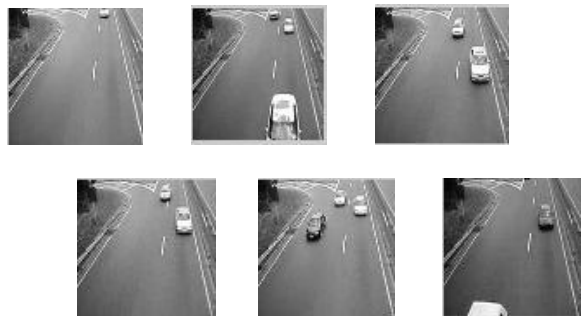

Figure 6. Watermarked frames after embedding process

After applying the watermark extraction algorithm, the extracted watermark is shown below:

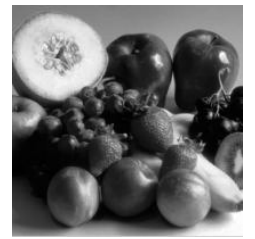

Figure 7. Watermark image after extraction process

\subsection{Quality Parameters}

The below section describes the quality parameters which are used to compare the quality of the original watermark and extracted watermark

\subsubsection{Mean square error}

The larger the value of the MSE implies the quality of the image is poor [18]. It can be defined as shown below:

$$
M S E=\frac{1}{m n} \sum_{i=0}^{m-1} \sum_{j=0}^{n-1}[I(i, j)-K(i, j)]^{2}
$$

where, $I$ is the original data and $K$ is the data after error addition.

\subsubsection{Peak signal to noise ratio}

It is a quality parameter that helps in identifying the distortion in the data after introduction of error. Firstly, the mean square error is computed which is used as an input to calculate psnr value. The smaller the value of PSNR implies the quality of image is poor [18]

The formulae are described below:

$$
M S E=\frac{1}{m n} \sum_{i=0}^{m-1} \sum_{j=0}^{n-1}[I(i, j)-K(i, j)]^{2}
$$

where, $\mathrm{I}$ is the original data and $\mathrm{K}$ is the data after error addition.

The PSNR can be calculated as:

$$
\begin{aligned}
\text { PSNR } & =10 \cdot \log _{10}\left(\frac{M A X_{I}^{2}}{M S E}\right) \\
& =20 \cdot \log _{10}\left(\frac{M A X_{I}}{\sqrt{M S E}}\right) \\
& =20 \cdot \log _{10}\left(M A X_{I}\right)-10 \cdot \log _{10}(M S E)
\end{aligned}
$$

where, MAX denotes the maximum value of image pixel. 


\subsubsection{Maximum Difference}

The larger the value of maximum difference implies the quality of the image is poor [18]. It can be expressed as follows:

$$
M D=\operatorname{Max}\left[\left|x(m, n)-x^{\wedge}(m, n)\right|\right]
$$

\subsubsection{Normalized absolute error}

The larger the value of the NAE, implies the quality of the image is poor [24]. It can be expressed as follows:

$$
N A E=\sum_{m=1}^{M} \sum_{n=1}^{N}\left|x(m, n)-x^{\wedge}(m, n)\right| / \sum_{m=1}^{M} \sum_{n=1}^{N}|x(m, n)|
$$

The table below shows the experimental results obtained based on the quality parameters used and the analysis of using various $\mathrm{db}$ wavelets.

Table 3. DB wavelet analysis using quality parameters

\begin{tabular}{|c|c|c|c|c|}
\hline $\begin{array}{l}\text { Wavelet } \\
\text { Name }\end{array}$ & MSE & PSNR & MD & NAE \\
\hline $\mathrm{db} 1$ & 0.255 & 14.1734 & 1.1918 & 0.9999 \\
\hline $\mathrm{db} 5$ & 0.2547 & 14.1857 & 1.0243 & 0.9999 \\
\hline $\mathrm{db} 9$ & 0.2547 & 14.1859 & 1.0247 & 0.9999 \\
\hline $\mathrm{db} 13$ & 0.2547 & 14.186 & 1.026 & 0.9999 \\
\hline $\mathrm{db} 17$ & 0.2547 & 14.186 & 1.0272 & 0.9999 \\
\hline $\mathrm{db} 21$ & 0.2547 & 14.1861 & 1.0272 & 0.9999 \\
\hline $\mathrm{db} 25$ & 0.2547 & 14.186 & 1.0257 & 0.9999 \\
\hline $\mathrm{db} 29$ & 0.2547 & 14.186 & 1.0254 & 0.9999 \\
\hline $\mathrm{db} 33$ & 0.2547 & 14.186 & 1.0245 & 0.9999 \\
\hline $\mathrm{db} 37$ & 0.2547 & 14.186 & 1.0259 & 0.9999 \\
\hline $\mathrm{db} 41$ & 0.2547 & 14.1861 & 1.028 & 0.9999 \\
\hline $\mathrm{db} 45$ & 0.2547 & 14.1861 & 1.0278 & 0.9999 \\
\hline
\end{tabular}

A comparative result of all the quality parameters for $\mathrm{db}$ wavelet analysis is graphically shown below:

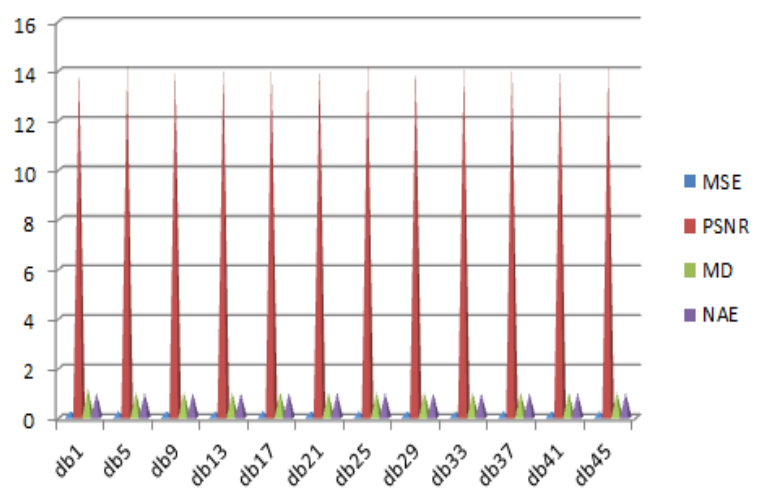

Figure 8. Comparison using all quality parameters

\section{CONCLUSION}

Watermarks can be of the form images, text, binary logos, signatures, and numbers. They are used for storing information about the copyright owner, source of data, and authentic users. Watermarking technique plays useful role in many areas such as intellectual and copyright protection, authenticity check, fingerprinting applications, and can also be used in pan card details, employee id cards etc. In the project work, video watermarking technique has been shown highlighting comparative analysis of $\mathrm{db}$ wavelets based on different quality parameters. Each of the $\mathrm{db}$ wavelets is applied on the randomly selected frames from the input coloured video using random number that works as a key for the proposed extraction algorithm. It is shown that not all $\mathrm{db}$ wavelets support watermarking scheme. Out of 45 wavelets, $12 \mathrm{db}$ wavelets were applicable for watermarking. The original watermark image and the extracted watermark image are then used as the basis against various quality parameters to check if the imperceptibility of the watermark is retained after watermark extraction. The proposed watermarking scheme is imperceptible against various quality parameters such as Peak-signal-to-noise ratio, Mean-square error, maximum difference, and normalized absolute error.

\section{FUTURE SCOPE}

Digital watermarking is the domain where lot of research is ongoing and much scope is there since it is a very popular and efficient technique to protect the copyright information and intellectual property of digital asset that is widespread over the internet medium. In our work, detailed analysis and evaluation of $\mathrm{db}$ wavelets has been showcased in an effective manner. Huge scope for further research work with the utilization of these $\mathrm{db}$ wavelets can be still present. The discussed watermarking scheme has been analyzed for various different $\mathrm{db}$ wavelets for video frames. These wavelet analyses can be carried further and can give good results for digitized multimedia such as audio. Security aspect can also be taken into consideration by making use of transforms such as Arnold transform that can cater to the security requirements of the videos widespread over internet. Also, the watermark can be compressed or encrypted before embedding inside the digital data for security enhancement. Instead of using DWT, complex wavelet transform can be used to enhance robustness and security of data. From future aspects, further more work can be done for enhancing the values of various quality parameters used. The $\mathrm{db}$ wavelets comparison can also be given considering different sizes, resolution of frames used.

\section{REFERENCES}

[1]Yang Qianli, Cai Yanhong, "A Digital Image Watermarking Algorithm Based on Discrete Wavelet Transform and Discrete Cosine Transform", 978-1-46732108-2/12/\$31.00 @2012 IEEE

[2]Mehran Andalibi, Damon M. Chandler, "Digital Image Watermarking via Adaptive Logo Texturization", IEEE TRANSACTIONS ON IMAGE PROCESSING, VOL. 24, NO. 12, DECEMBER 2015

[3]Yueh-Hong Chen, Hsiang-Cheh Huang, "Genetic Watermarking Based on Wavelet Packet Transform", 978-0-7695-3745-0/09 \$25.00 @ $\quad 2009$ IEEE DOI 10.1109/HIS.2009.59

[4]Hassen Seddik, Mounir Sayadi and Farhat Fnaiech, "A New Blind Image Watermarking Method Based on Schur transformation", 978-1-4244-4649-0/09/\$25.00 (02009 IEEE

[5]Saied Amirgholipour Kasmani, Ahmadreza Naghsh-Nilchi "A New Robust Digital Image Watermarking Technique Based On Joint DWT DCT Transformation", Third 2008 International Conference on Convergence and Hybrid Information Technology, 2008 IEEE. 
[6] Gunjan Malik and Tarun Kumar, "Analysis of Watermarking Techniques", IJCA, Volume 138 - No.10, March 2016.

[7]S. Vongpradhip and S. Rungraungsilp, "QR code using invisible watermarking in frequency domain," in Proc. 9th Int. Conf. ICT Knowl. Eng., 2012, pp. 47-52.

[8]S. Bhalerao, M. Mehta, N. Dubey, and M. Bhatele, "Digital watermarking of medical images," in Proc. India Seminar Biomed. Eng., 2012, pp. 111-116.

[9]Cheng-Han Yang,Hui-YuHuang,Wen-Hsing Hsu, "An adaptive video watermarking technique based on DCT domain", 978-1-4244-2358-3/08/\$20.00 @ 2008 IEEE

[10]Mr. Amit M Joshi, Dr. R.M.Patrikar, Dr. Vivekanand Mishra, "Design of Low Complexity Video Watermarking Algorithm based on Integer DCT", 978-14673-2014-6/12/\$31.00 @2012 IEEE

[11]Wei Wang, Aidong Men and Xiaobo Chen, "Robust Image Watermarking Scheme Based on Phase Features in DFT Domain and Generalized Radon Transformations", 978-1-4244-4131-0/09/\$25.00 @2009 IEEE

[12]I. Cox, J. Kilian, T. Leighton, T. Shamoon, "Secure spread spectrum watermarking for multimedia", IEEE Trans. Image Process. No. 6, Vol. 12, June 1997, pp.1673-1687.

[13]Jeril George, Satishkumar Varma, Madhumita Chatterjee, "Color Image Watermarking using DWT-SVD and
Arnold Transform", 2014 Annual IEEE India Conference (INDICON)

[14]Santi P. Maity,Malay K. Kundu, "An Image Watermarking Scheme using HVS Characteristics and Spread Transform", Proceedings of the 17th International Conference on Pattern Recognition (ICPR'04) 10514651/04 \$ 20.00 IEEE

[15] Nisreen I. Yassin, Nancy M. Salem and Mohamed I. El Adawy, "Entropy Based Video Watermarking Scheme Using Wavelet Transform and Principle Component Analysis", 978-1-4673-4810-2/12/\$31.00 (O2012 IEEE

[16]S. A. Mostafa, A. S. Tolba, F. M. Abdelkader, and H. M. Elhindy, "Video Watermarking Scheme Based on Principal Component Analysis and Wavelet Transform", International Journal of Computer Science and Network Security, vol. 9, no. 8, August 2009.

[17]Nilanjan Dey, Anamitra Bardhan Roy, Poulami Das, Achintya Das, Sheli Sinha Chaudhuri, "DWT-DCT-SVD based IntravascularUltrasound video Watermarking", 2012 World Congress on Information and Communication Technologies, 978-1-4673-48058/12/\$31.00 c 2012 IEEE

[18] V.S Vora, Prof. A.C Suthar, Y.N.Makwana, S.J Davda, "Analysis of Compressed Image Quality Assessments", Published in International Journal of Advanced Engineering \& Application, Jan. 2010. 